(i)

\title{
Editorial
}

\section{Doctor as Teacher and Books and Journals as Educator}

The word "Doctor" derives from Latin "Docere" which means "to teach" (in fact all words 'doctor', 'docile', 'docent', 'document' and 'doctrine' derive from the Latin word "docere" in one way or the other and describe the quality of a teacher). Unexpectedly the word shows up in the roots of the word 'docile' is easy to teach and also the word 'document', which originally the thing from which one took information that was to be taught. Therefore, it is the responsibility of all doctors (with medical qualifications) to pass on their knowledge skills for the good of the profession in the future. This could also be considered as a "duty of care" which ensures tomorrow's doctors are well trained and knowledgeable as possible, and helps to ensure best possible care for tomorrow's patients as well.

Within minutes of consulting a new patient, a doctor knows what caused the problem he/she has and also knows what you have to tell them and how to treat $\mathrm{him} / \mathrm{her}$. When we engage a patient in the process of listening and explaining to $\mathrm{him} /$ her with an attitude of compassion and empathy, it develops a bond of trust. With engaged patients, medical problems can be diagnosed swiftly and treated with higher success rate. Illness can be prevented and emergency department usage can be reduced. Care can be personalized to improve outcomes that matters to providers and patients as well. In the process, patient engagement can indeed perform miracles : it can combat voracious healthcare costs.

The attitude of doctors towards profession influences to a large extent a number of aspects of clinical competence. Their attitude towards the patient is particularly important as it determines the quality of communication. There is a reason to believe that a number of practising clinicians have not acquired the appropriate attitude to their patients and the skills in communicating that this entails inspite of specific undergraduate medical education programmes. This specially warrants more and different attention to this subject by medical colleges than is actually the case. This also requires introduction of syllabi and curricula which help in developing attitudes between doctor-centred, disease-oriented, patient-centred and problem-oriented basis of studying, teaching and evaluating individual attitudes of medical students.

Besides patient engagement, the other thing which matters in making a knowledgeable and competent health care provider is evidence-based medical 
knowledge, just as being a good doctor is far more than merely being a good clinician. It must involve fostering of a critical awareness- a critical consciousness of self, others, and the world and a commitment to addressing issues to societal relevance in health care. On way of doing is fostering a habit of reading medical books and journals of one's interest with the aim to serve the society in a better way. It also involves not only reading but contributing to the current medical literature by one's own medical experiences and researches.

Now a look at the contents of the present issue of the Annals : spine-related disorders and deformities are among the most frequently encountered problems in clinical medicine. Near about $80 \%$ of the population, at some point in life suffer from low back pain that causes $1-2 \%$ of such population to become morbidly disabled. The need for care of these patients has led to an array of treatment options, including medications, manipulative care, interventional spine procedures, and a number of surgical approaches. There are several emerging interventional innovative spine procedures but the role that many of these play in spine care is unclear. Perhaps the best way to address the future is to examine the past.

Oral cancer has a tendency to be detected at late stage but an early detection of such cancer is an important aspect to reduce the burden of this devastating disease. The 5-year relative survival rates for cancer of oral cavity and pharynx are $83 \%$ for cancer that has not spread, $62 \%$ for cancer that has spread to nearby lymph nodes and $38 \%$ for cancer that has spread to distant parts. On the other hand, study of cervical cancer, the fourth-most common cause of cancer and death from cancer in women after the breast, lung and colorectal cancers. The 5-year survival rate for all women with cervical cancer is $68 \%$. This paper attempts to show its multi-factorial causation, potential for prevention and successful treatment if diagnosed early. The study of its epidemiology through National Cancer Registry Programme (NCRP) and a more comprehensive and larger programme called National Programme for Prevention and Control of Cancer, Diabetes, Cardiovascular Disease and Stroke (NPCDCS) are important keys to success to address common risk factors and treatment strategies for reducing the scourge of such diseases in the affected population. The reaching goal of health care delivery must be for achieving high value for patients, with value defined as the health outcomes achieved per expenditure made. This goal is what matters for patients and if value improves, patients, payers, providers, and suppliers can all benefit while the economic sustainability of the health care system increases. Value in health care remains largely unmeasured and misunderstood. Both scientific papers related to cervical cancer and oral cancer emphasize this point more relevantly. To add to the variety, the rest two articles are related to medical education. How innovations in teaching of complex topics of medical anatomy make the matter 
(iii)

simple to understand by using physical models of middle ear, which as such seems to be difficult when accessed through the cadaveric study and text book reading, has been explained in one paper. Whereas the other paper compares the merits of students' evaluation through carefully designed short-answer questions with the multiple-choice questions in the area of same topic.

The Annals, the official publication of the National Academy of Medical Sciences is a platform which provides good opportunity to medical fraternity of specialists to not only read but also publish and share their experiences and researches to emphasize the role and current status of research in India for the young minds by its rich contents. The present issue is an example of uniformity in diversity as it contains articles with a blend of variety of medical issues by doctors who are "docere" in real sense of the term.

Dr. Mukund S Joshi

President, NAMS 